\title{
L'hygroma brucellique: l'aspect clinique caractéristique de la brucellose bovine au Rwanda-Burundi
}

\author{
par D. THIENPONT, M. VANDERVELDEN, P. FAGARD ef J. MORTELMANS.
}

\section{INTRODUCTION}

BURNET a écrit que, lors de la brucellose, il y a en général beaucoup plus d'infectés que de malades. Une telle thèse se confirme, non seulement en Europe, mais aussi et surtout dans les élevages des pays tropicaux. L'aspect clinique y est si vague que les éleveurs autochtones ignorent la maladie puisqueles symptômes caractéristiques, l'avortement isolé ou l'avortement épizootique, font génćralement défaut. Si la brucellose n'est pas connue sous sa forme classique par l'éleveur, celui-ci désigne cependant par un nom spécifique, «AMAKOLE», les bursites et les tendovaginites. Un phénomène similaire est signalé par CAMARA (1948) (1) citant pour I'A. O. F. le terme «BAKALE》; par SAQUET (1955) (2) et par PERREAU (1956) (3) donnant le nom «BAKELE » au Tchad de même que BLANCHARD et SINALY COULIBALY (1954) (4) en Haute Volta.

Ces trois termes doivent être considérés comme synonymes de la brucellose dans son extériorisation sous-cutanée. Tous les manuels donnent, lors de l'étude des symptômes ef des lésions de la brucellose, une description sommaire des hygromas brucelliques. HENNING (1956) (5) signale que des animaux atteints de brucellose, souffrent parfois d'hygromas, de synovites ef d'arthrites. MANNINGER, MAREK et MOCSY (1959) (6) citent les mêmes symptômes et reprennent les observations de THOMSEN sur les abcès sous-cutanés provoqués par Brucella abortus Bang.

VANDER WATH (1939) (7) au Transvaal, dans une étude sur le diagnostic différentiel de l'ar-

Rev. Elev, méd. vét. Pays trop., 1961, 14, no 3.

Reçu pour publication : juin 1961. thrite chronique des bovidés, altire l'attention sur les bursites et les arthrites d'origine brucellique d'où il isole les microbes spécifiques. II confirme ainsi les études de BERGMAN ef AGREN (1923) (8) et celles de BOYD, DELEZ et FITCH (1930) (9). Plusieurs autres auteurs ont également constaté les hygromas et les synovites sans cependant confirmer le diagnostic par des culfures bactériologiques : BLANCHARD et coll. (1954) (4) ; PERREAU (1956) (3); CAMARA (1948)(1); DAFAALA ET KHAN (1958) (10). En Allemagne DIETZ, NAGEL ef TURICH (1959) (11) isolent le bacille de Bang dans 40 c̀ 50 p. 100 des cas de bursite, de tendovaginite et d'arthrite des bovidés.

THIENPONT et coll. (1958) (12), lors d'une large prospection dans la préfecture d'Astrida (Rwanda-Burundi), constatent, d'une part l'existence de la brucellose, d'autre part la présence de nombreux hygromas desquels ils isolent aussi le bacille spécifique dans 60 p. 100 des cas.

COID et VAUGHAN (1957) (13), après avoir d'abord signalé l'existence en Angleterre d'hygromas sur du bétail avec agglutination positive pour brucellose, rapportent que l'incidence de l'hygroma carpien, chez le bétail infecté expérimentalement avec Brucella Bang, n'est pas, de façon significative, plus élevée que chez le bétail de contrôle non vacciné.

KELLER (1951) (14) isole le bacille de Bang de trois veaux atteints de polyarthrite.

L'évolution de la brucellose humaine s'accompagne souvent aussi de bursites et de tendovaginites: HOFMANN (1952) (15) en Allemagne; TUSZKIEWICZ et coll. (1958) (16) en Pologne; MANSON BAHR (1956) (17) en Afrique Orientale et FAGARD et VAN BALEN (1961) (18) au Rwanda-Burundi. 


\section{L'EXISTENCE DE LA BRUCELLOSE AU RWANDA-BURUNDI}

Jusqu'en 1958, le Rwanda-Burundi ne disposait pas de donriées précises sur l'imporlance de la brucellose dans le pays. Le Laboratoire vétérinaire de Kisenyi et, plus tard, celui d'Astrida, avaient posé à plusieurs reprises, pour des cas isolés, le diagnostic de brucellose.

En 1950, SCHOENAERS (1950) (19) lors d'un coup de sonde sur le bétail du nord-ouest du Rwanda, fait ressortir le rapport entre la brucellose et l'avortement: ses chiffres laissent prévoir une incidence insoupçonnée de la maladie. A la clinique de l'Ecole d'assistants vćtćrinaires d'Astrida, l'un de nous attira l'attention sur l'existence chez le bétail de multiples hygromas aux endroits les plus divers du corps.

L'enquête de 1958. - THIENPONT (1958) (12) a révélé que, dans la préfecture d'Astrida, le pourcentage d'infection varie d'une région à l'autre. Contrairement à ce qu'on pourrait supposer, c'est dans les régions les plus chaudes que le pourcentage de réactions d'agglutination positives se révèle le plus élevé. Dans les régions froides et humides, à une altitude de 1900 à 2300 mètres, le pourcentage est de 1,8 d̀ 4,8 p. 100; dans les régions plus basses et plus chaudes, à une altitude de 1500 à 1700 mètres, il varie entre 7,78 et 19,75 p. 100.

Au cours des années suivantes, le laboratoire vétérinaire d'Astrida confirme l'existence de la brucellose dans toutes les préfectures et tous les territoires du Rwanda-Burundi (1959-1960) (20).

\section{L'EXISTENCE DE L'HYGROMA BRUCELLIQUE}

Comme d'autres auteurs déjà signalés dans l'introduction, nous avons remarqué, sur le bétail du Rwanda-Burundi, l'existence de bursites, de tendovaginites, d'arthrites et de périarthrites. Ces animaux apparemment sains ne semblent pas souffrir de ces lésions dans leur état général. Mais les animaux amaigris, sous l'influence d'une saison sèche prolongée ou souffrant d'une des multiples maladies tropicales parasitaires, et atteints d'hygromas, paraissent d'autant plus fortement défigurés.
Lors d'une nouvelle enquête, menée dans la préfecture d'Astrida, sur 1351 bovidés jeunes ef adultes des deux sexes, nous découvrons 78 animaux atteints de un ou de plusieurs hygromas. Dans cette étude, nous classons sous le terme hygroma les bursites, les tendovaginites, les périarthrites, les arthrites et les abcès sous-cutanés d'origine brucellique.

Chez un animal sain, la bourse muqueuse normale est située dans le tissu conjonctif souscutanć, où la pcau rccouvrc les parties osseuses saillantes. Ces petites cavités renferment une infime quantité d'un liquide visqueux et transparent.

Lors d'une inflammation, qu'elle soit d'origine mécanique ou infectieuse, la paroi interne de la bourse muqueuse présente l'apparence d'une vraie muqueuse. Elle sécrète une quantité anor male de liquide etelle est le siège d'une inflammation. Lors d'une infection par Brucella abortus, on constate une inflammation séro-fibrineuse d évolution lente', mais progressive et envahissante. La membrane interne sécrétante se développe et s'épaissit, forme des proliférations internes et des brides, s'étend vers la périphérie, envahit le tissu sous-cutané et augmente progressivement de volume par l'accumulation d'e l'exsudat inflammatoire. A la périphérie, il se produit une réac. tion fibreuse. La paroi conjonctive kystique est parfois assez épaisse et peut même atteindre trois à quatre millimètres. L'examen du contenu hygromique se fait par ponction. La couleur du liquide est variable; elle peut être opalescente ou hémorragique, mais elle revêt généralement une teinte citrine ou jaunatre. Le liquide peut être transparent ou chargé de grumeaux fibrineux de grandeur variant de celle du grain de riz cuit à celle d'une masse de forme irrégulière grande comme une noisette. Selon l'âge et l'évoIution du phénomène inflammatoire, la consislance peut être aqueuse, visqueuse, purulente, caséeuse ou crayeuse. La quantité de liquide est fort variable. Des petits hygromas, on peut d̀ peine soutirer un ou deux centimètres cubes, mais des hygromas volumineux contiennent parfois plus d'un litre de liquide. Un hygroma localisé entre les deux ligaments de la nuque, constaté chez une vache adulte et souffrant depuis déjà plus de deux ans de cețe affection, contenait plus de vingt litres de liquide. 


\section{NOMBRE D'HYGROMAS}

Lors de l'enquête, on établit pour chaque animal une fiche signalant: le sexe:" l'age et la robe ; les antécédents c'est-d̀-dire le nombre de vêlages et d'avortements; la présence, le nombre ef la localisation des hygromas; les réactions sérologiques et, éventuellement, le résultat de la culture du produit de ponction de l'hygroma.

Parmi les 78 bêtes porteuses d'hygromus, nous comptons 48 animaux avec un hygroma et 30 autres avec deux ou plusieurs hygromas qui se répartissent ainsi : 21 bêtes avec deux hygromas, 2 bêtes avec trois hygromas, 4 bêtes avec quatre hygromas et 1 bête avec respectivement six, sept ef huit hygromas.

Au total sur les 78 cas nous avons dénombré et excminé 133 hygromas.

Citons, à titre d'information, la localisation des hygromas chez les trois dernières bêtes, complètement défigurées par ces tuméfactions éparpillées sur tout le corps.

Vache avec 6 hygromas: aux deux genoux, aux deux jarrets et un de chaque côté à l'angle externe de la hanche.

Vache avec 7 hygromas: aux deux genoux, aux deux jarrets, au boulet gauche et droit des pattes antérieures et à l'angle externe de la hanche à gauche.

Vache avec 8 hygromas: au genou gauche, aux deux jarrets, deux aux tubérosités ischiales, à l'angle externe de la hanche à droite, au niveau du chanfrein et au grasset gauche.

Les vaches adultes, âgées de 8 à 12 ans, sont plus souvent atteintes que les jeunes. Nous avons cependant relevé de multiples hygromas chez les génisses mais il est exceptionnel de constater ces mêmes lésions chez les faurillons, les taureaux et les bœufs. Les raisons en sont multiples: la moindre sensibilité du mâle à la brucellose, le nombre peu élevé de taureaux et de taurillons examinés et, en dernier lieu. l'éleveur indigène, tenant à ccur l'esthétique d'un reproducteur, élimine pour la boucherie le taureau atteint d'hygroma.

\section{LA LOCALISATION DES HYGROMAS}

II est étonnant que la plupart des auteurs décrivent surtout les hygromas au niveau de l'articulation carpienne, tels VANDER. WATH (7) DIETZ et coll. (11), COID et coll. (13). PERREAU (3) cependant les signale, sans spécifications, aux pattes antérieures et postérieures.

Nous ne pouvons confirmer leurs observations, car les 133 hygromas se répartissent ainsi : 60 hygromas constatés sur les pattes postérieures, 40 hygromas sur les pattes antérieures et 33 hygromas à des endroits divers (tableau 1).

II n'est donc pas question d'admettre des lieux de prédilection pour les hygromas, mais un phénomène anatomique frappe quand même: le jarret et le genou sont le plus tréquemment atteints.

TABLINJ 1 - Localization des hygromas

\begin{tabular}{|c|c|c|}
\hline Membres postérieurs & Heabres antérieurs & Divers \\
\hline 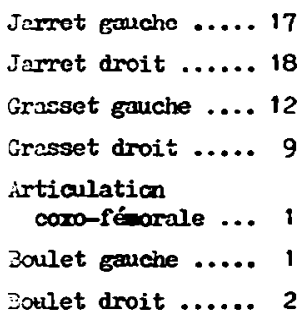 & 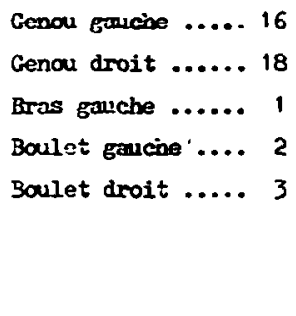 & 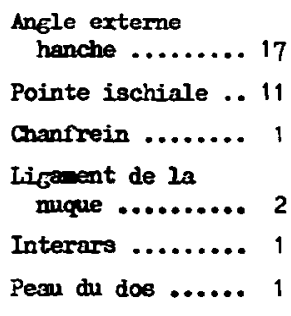 \\
\hline 60 & 40 & 33 \\
\hline \multicolumn{3}{|c|}{ Total $=133$} \\
\hline
\end{tabular}




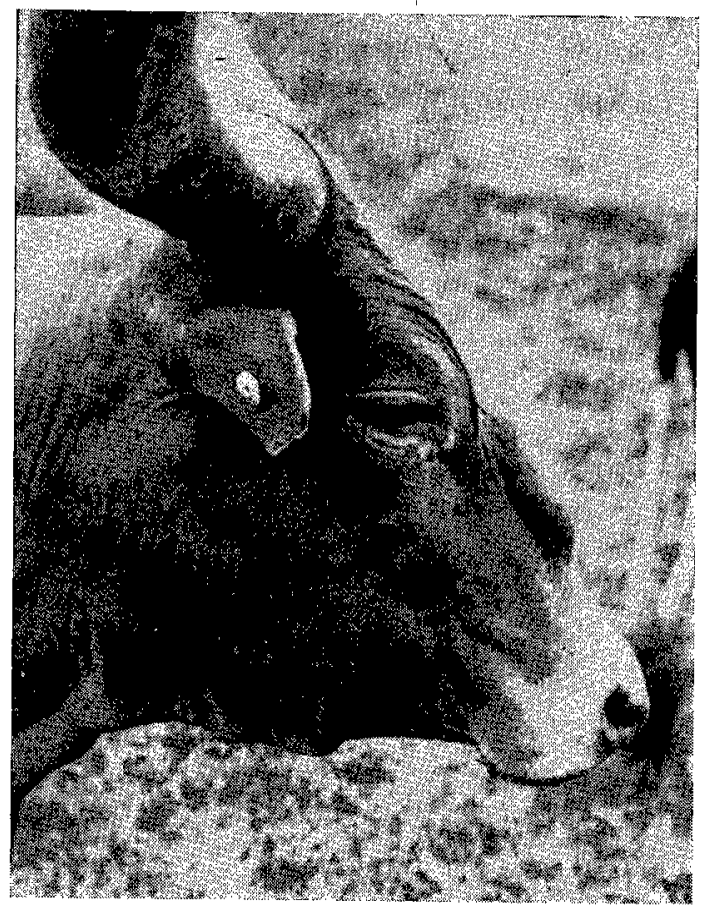

Phot. 1. - Hygroma au niveau du chanfrein.

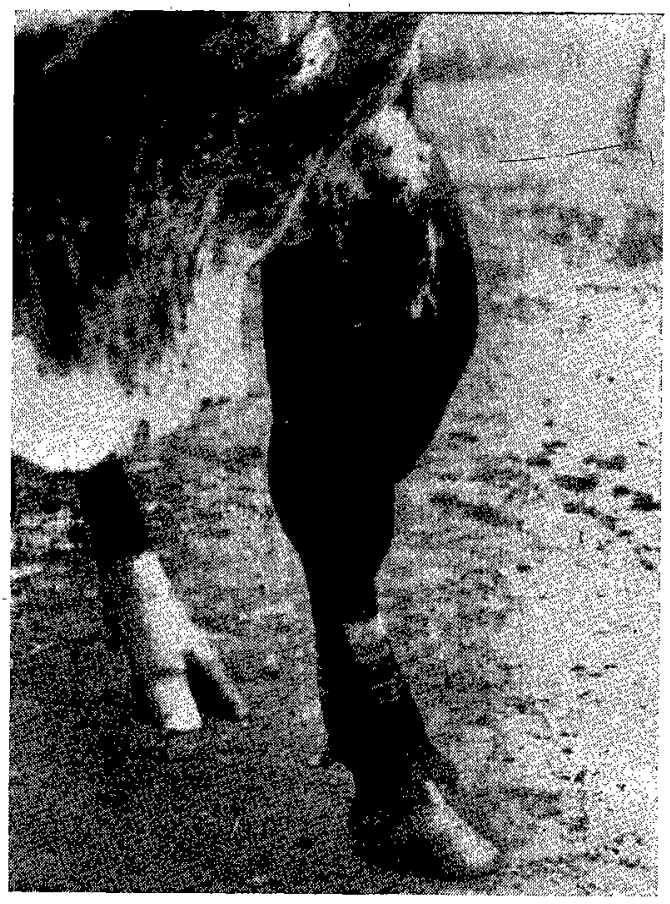

Phot. 2. - Enorme hygroma de l'avant-bras.

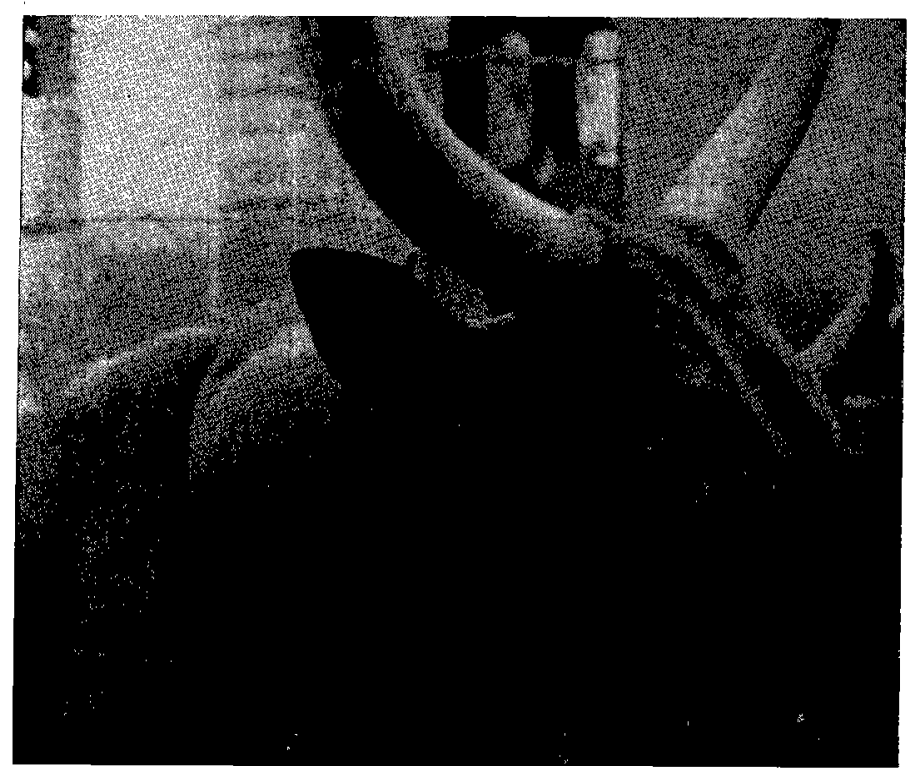

Phot. 3. - Hygroma entre les deux ligaments de la nuque. 


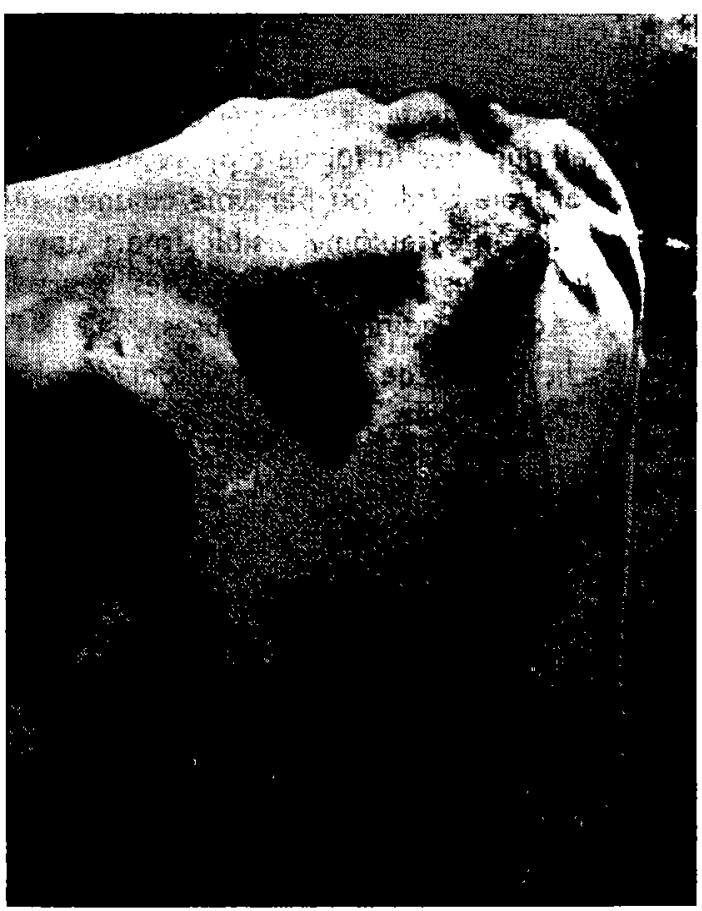

Phot. 4. - Hygroma au niveau de l'angle externe de la hanche (2) et des vertèbres sacrées.

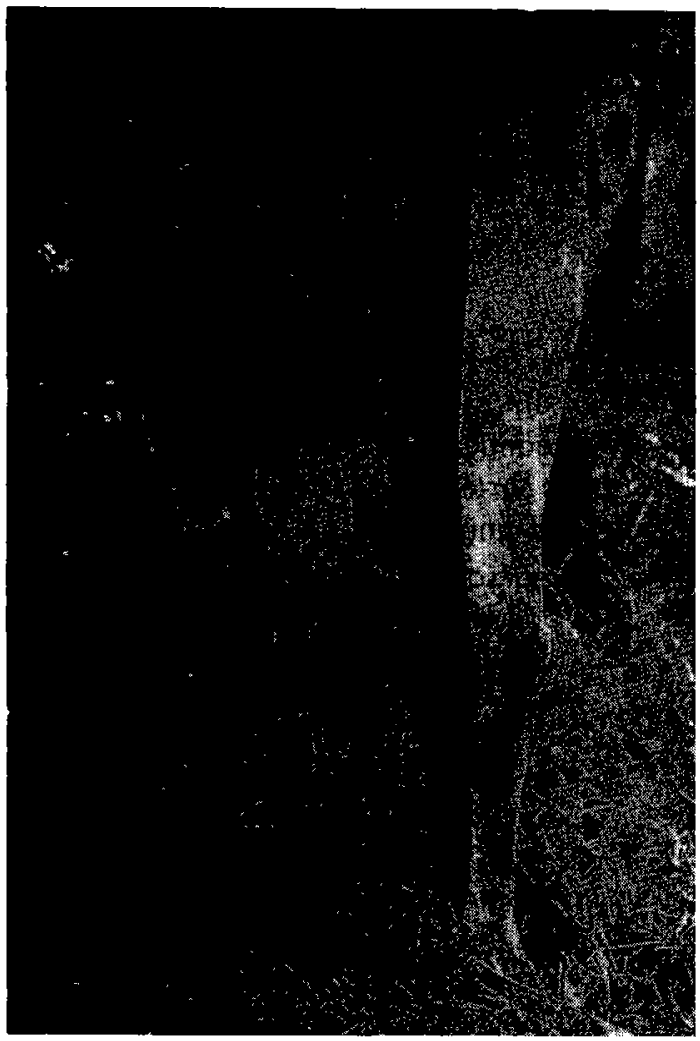

Phot. 5. - Hygromas au niveau des deux jarrets et du grasset gauche.

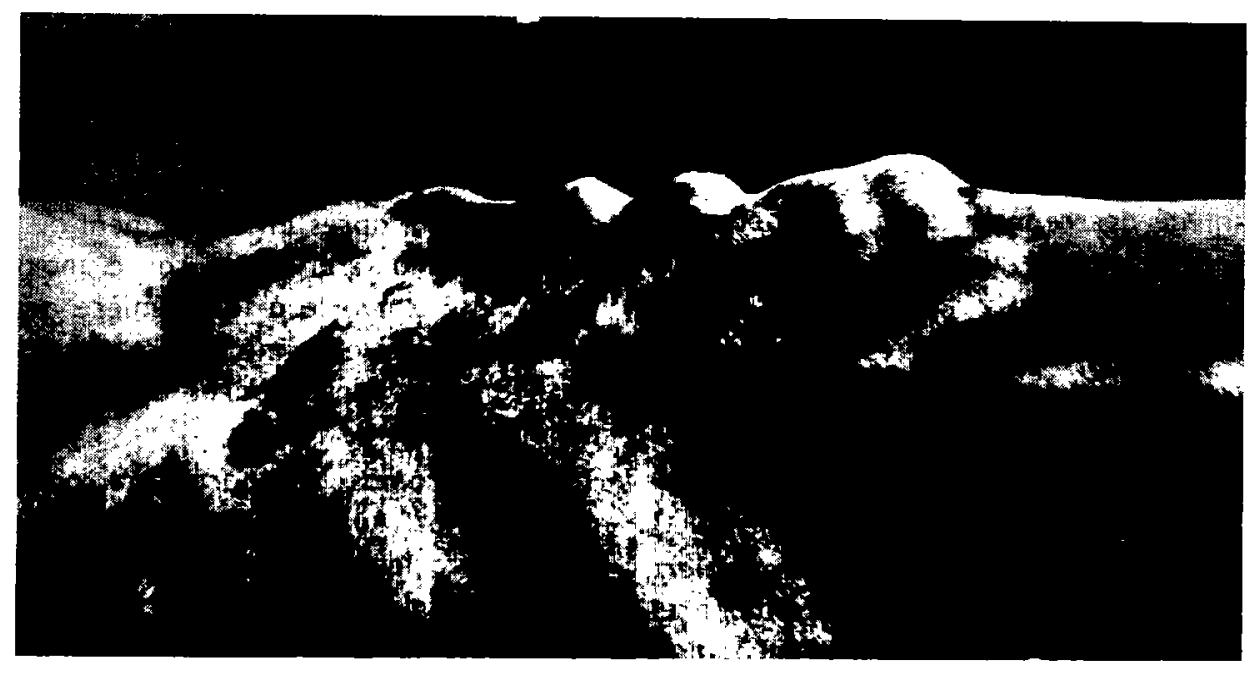

Phot. 6. - Abcès brucelliques sous-cutanés. 
Quelques-unes de ces localisations sont exceptionnelles et, à notre avis, n'ont jamais été décrites. Au niveau du chanfrein, il n'existe pas de bourse muqueuse. L'hygroma se prolonge sur la partie dorsale du chanfrein et se présente comme une tuméfaction indolore, molle et bien délimitée (photo 1).

L'hygroma, localisé entre les deux ligaments de la nuque, a déjà été mentionné à cause de son énorme développement (photo 3). L'animal atteint a un aspect presque monstrueux par l'énorme gonflement de la région cervicale; la peau est fortement tendue et les mouvements du cou sont limités et raidis.

L'hygroma sous-cutané au niveau du dos est constitué par de multiples petits abcès brucelliques (photo 6).

\section{L'AGE DES ANIMAUX ATTEINTS D'HYGROMA}

Il est nécessaire d'en référer à la monographie pastorale du Rwanda-Burundi (ADAMANTIDIS 1956) (21) et à l'élevage en Urundi (MATHIEU 1960) (22) pour pouvoir se rendre compte des pratiques d'élevage dans ces pays. Nous devons seulement insister ici sur l'extrême rusticité ef la grande tardivité du bétail local. Les génisses sont mises au taureau à l'âge de quatre ans et exceptionnellement plus tôt. Mais une génisse impubère de cinq ans ne doit pas être considérée comme un fait extraordinaire. La possibilité d'infection avant la puberté est d'autant plus grande, les génisses et les vaches vivantet logeant ensemble dans le kraal et broutant sur les mêmes pâturages.

La longivité des reproductrices n'est pas très élevée: rares sont les vaches qui ont plus de quinze ans.

Le rythme de reproduction est très lent, en moyenne une vache donne un veau tous les deux ans.

Parmi les 78 bêtes avec hygromas, nous relevons 8 génisses et 70 vaches.

A notre avis, nous constatons pour la première fois des hygromas brucelliques chez des génisses impubères et chez des génisses n'ayant jamais été saillies et dont l'âge varie entre un an et demi à quatre ans.
Ceci est un phénomène inconnu en Europc parmi le bétail précoce. Mais au RwandaBurundi et dans les pays voisins, l'élevage indigène comprend exclusivement $d u$ bétail tardif. Au cours de la longue période préparatoire à la reproduction, le jeune bétail a toutes les possibilités de s'infecter et d'extérioriser l'affection brucellique sous la forme d'hygromas. L'infection, par voie orale ou par voie cutanée, ne provoque aucun symptôme visible, mais après une bacillémie, les bacilles gagnent les bourses muqueuses ou les gaines tendineuses.

Les vaches âgées de 7 à 12 ans sont les plus atteintes. Nous avons examiné dans le lot de 70 vaches : 8 bêtes de 7 ans, 15 de 8 ans, 17 de 9 ans ef 12 de 10 ans.

\section{DATE DE L'APPARITION DES HYGROMAS}

II n'est pas facile d'établir une anamnèse correcte d'après les déclarations de l'éleveur indigène qui ignore parfois les renseignements demandés ou qui ment par méfiance.

Nos données sont donc nécessairement incomplètes, mais elles donnent des informations assez instructives pour situer le problème.

En résumé, on peut admettre que l'hygroma apparaît à n'importe quel moment. Tel est le cas d'une génisse de un an et demi déjà atteinte depuis quatre mois; d'une autre génisse de 3 ans atteinte depuis un an et demi; d'unc vache de 6 ans atteinte depuis deux ans c'est-à-dire avant la mise au taureau.

Chez les vaches, les hygromas apparaissent généralement après un avortement ou après un vêlage normal. Tel est le cas d'une vache de 12 ans qui a avorté à la première gestation ef qui, par après, a donné quatre veaux vivants: les hygromas sont apparus après le $3^{e}$ vêlage normal à l'âge de 10 ans.

Les hygromas n'apparaissent pas tous en même temps. Au cours des années, des vêlages ef des avortements éventuels mais rares, on constate leur apparition. Tel est. le cas d'une vache de 11 ans, n'ayant jamais donné de veau vivant, avec 4 hygromas : après chaque avortement un nouvel hygroma s'est formé. 


\section{DISCORDANCE DES RÉSULTATS BACTÉ- RIOLOGIQUES ET SÉROLOGIQUES}

Pour faire un diagnostic aussi précis que possible et en vue de confronter les différents résultats, nous avons pris du sang de chaque animal et, de chaque hygroma par ponction, du liquide pour la culture bactériologique (tableau II).

TABLEAI II - Résultats:culture de l'hyEToma et agclutination du sang

\begin{tabular}{|c|c|c|c|}
\hline $\begin{array}{r}\text { Prćsence } \\
\text { d'hygroma }\end{array}$ & $\begin{array}{c}\text { Souche } \\
\text { isolée }\end{array}$ & $\begin{array}{c}\text { Asglutination } \\
\text { du sang }\end{array}$ & Nombre \\
\hline+ & + & + & 29 \\
+ & + & - & 14 \\
+ & - & + & 11 \\
+ & - & - & 24 \\
\hline
\end{tabular}

En analysant ces résultats, nous relevons d'abord la première possibilité qui est d'ailleurs la seule logique : 29 souches de Brucella provenant de 29 bêtes avec hygromas dont l'agglutination du sang est positive.

La deuxième possibilité est anormale car, dans 14 cas, on isole une souche de Brucella de l'hygroma, mais l'épreuve sérologique du sang est négative chez ces animaux. Sans la ponction de l'hygroma, ces 14 bêtes auraient pu être considérées comme non atteintes de brucellose, du moins au moment de notre examen.

Dans les 35 autres cas provenant de 11 bêtes avec agglutination positive et 24 bêtes avec agglutination négative, on n'isola pas des Brucella.

La question se pose si on peut, dès lors, les considérer comme des hygromas brucelliques ou comme des hygromas d'origine mécanique et alors stériles ou d'origine microbienne variée. Dans cinq cas, on isole des microbes pyogènes, mais chaque fois, it s'agit d'hygromas traités par des pointes de feu qui ont peut-être provoqué une infection secondaire. Un hygroma enkysté peut certainement se stériliser spontanément et ainsi devenir négatif.

S'ily a une première discordance entre l'épreuve sérologique négative du sang ef la culture positive de l'hygroma, il y en a une seconde non moins importante : c'est l'existence d'un titre d'agglutination dans le liquide hygromıque qui ne correspond pas avec le titre du sang.

Dans 4 cas nous constatons les anomalies suivantes :

TABLEAU III

\begin{tabular}{|c|c|c|}
\hline $\begin{array}{c}\text { Ponciion de } \\
\text { I'hygrcma }\end{array}$ & $\begin{array}{c}\text { Azglutination } \\
\text { cu sang }\end{array}$ & $\begin{array}{c}\text { Agrlutination } \\
\text { du liquide } \\
\text { de l'hygroma }\end{array}$ \\
\hline Souche & $\frac{1}{40}$ & $\frac{1}{160}$ \\
Souche+ & 0 & $\frac{1}{320}$ \\
Souche + & $\frac{1}{40}$ & $\frac{1}{160}$ \\
Souche + & $\frac{1}{80}$ & $\frac{1}{40}$ \\
\hline
\end{tabular}

Le premier cas confirme la thèse qu'un hygroma peut se stériliser et contenir encore une grande quantité d'agglutinines. Dans I'hygroma, les agglutinines sont probablement élaborées sur place et diffusent éventuellement dans le courant sanguin (cas no 1, 2 et 3). Lors de bacillémies répétées, avec titre sanguin élevé, les agglutinines diffusent vers l'hygroma (cas no 4) et atteignent le même titre dans le sang et dans le liquide hygromique (constaté à deux reprises chez des animaux dont le titre sanguin et le titre hygromique furent de $1 / 640$ ).

La culture et la réaction sérologique du liquide de l'hygroma sont deux moyens de diagnostic utiles pour la brucellose.

\section{L'EXISTENCE DE L'HYGROMA ET DE L'AVORTEMENT}

Dans l'introduction, nous avons insisté sur le peu d'importance que l'éleveur attache à l'avortement, puisque ce dernier ne se présente pas sous la forme épizootique. Depuis des siècles, on a constaté ce fait et on l'explique par le mauvais sort ou les esprits défavorables. C'est pour cette raison qu'il est si difficile d'obtenir des renseignements précis par l'anamnèse.

Lors de l'enquête, les propriétaires ont déclaré que, sur les 1351 bêtes, 108 vaches ont une ou plusieurs fois avorté, soit près de 8 p. 100. Pour les 78 bêtes avec hygromas on nous a informé que 25 ont déjà avorté, soit plus de 32 p. 100. 
Nous mettons en relief la disproportion de ces deux pourcentages pour admettre qu'il existe une relation entre l'hygroma et l'avortement. Le premier, comme le second, sont des phénomènes consécutifs à l'infection brucellique.

\section{LE TRAITEMENT DE L'HYGROMA'}

En milieu indigène, l'hygroma est traité par des pointes ou des lignes de feu. Loin de provoquer une amélioration, il constitue généralement une porte d'entrée pour des infections secondaires localisées ou généralisées.

Avant d'avoir eu connaissance des travaux de DIETZ et coll. (II) nous injections dans l'hygroma une quantité de formol à 10 p. 100 de façon à atteindre dans le liquide une concentration de 1 p. 100 environ. Nous stérilisons ainsi in vivo un foyer inflammatoire extrêmement dangereux.
L'injection de formol provoque à la périphérie de l'hygroma un œdème modéré.

Après trois jours, on injecte dans l'hygroma du sulfate de cuivre en solution aqueuse à 10 p. 100 de façon à obtenir également une concentration de 1 p. 100. Sous l'effet du sulfate de cuivre, la paroi kystique de l'hygroma subit la nécrose ef un sillon disjoncteur se forme progressivement, séparant les tissus mortifiés des tissus sains.

Une semaine après l'injection du sulfate de cuivre, on ouvre au bistouri l'hygroma, dans la partie la plus déclive; à l'aide d'une pince de Museux, tout l'hygroma se laisse enlever. L'existence d'adhérences nécessite l'usage de cisecux. Après saupoudrage d'antibiotiques, on recoud la peau et on met, si cela est possible, un pansement compressif.

$$
\begin{aligned}
& \text { Ecole des assitants vétérinaires } \\
& \text { d'Astrida } \\
& \text { Directeur: Dr. D. Thienpont. }
\end{aligned}
$$

\section{RÉSUMÉ}

La brucellose existe partout au Rwanda-Burundi. Le pourcentage varie de région à région ef oscille entre 1 et 20 p. 100. L'hygroma doit être considéré comme le synonyme de la brucellose dans son extériorisation sous-cutanée. Par l'examen clinique où domine l'épreuve de la ponction, et par l'examen bactériologique et sérologique du liquide de ponction, le diagnostic est confirmé dans 60 p. 100 des cas. Lors d'une enquêtesur 1351 bovidés de race indigène d'éleveurs autochılones, 78 animaux sont atteints d'hygroma dont 48 avec un hygroma et 30 avec de deux à huit hygromas.

Sur les 78 bêtes, on dénombre 133 hygromas dont 60 aux membres postérieurs, 40 aux membres antérieurs et 33 à des endroits divers comme l'angle externe de la hanche, au niveau du chanfrein, entre les ligaments de la nuque et, sous forme d'abcès sous-cutanés, sur le dos.

Générulemenl, les vaches âgées de 7 à 10 uns sonl te plus souvent ulleintes, mais on conslale égulement des hygromas chez des génisses de 1 à 4 ans.

Les hygromas apparaissent à n'importe quel moment, mais généralement après un avortement ou un vêlage. Chez les génisses on les constate déjà avant la puberté. L'agglutination du sang peut être négative chez un animal porteur d'un ou plusieurs hygromas brucelliques. Le titre d'agglutination du sérum sanguin ne correspond pas toujours avec le titre d'agglutination du liquide hygromique. II existe une relation entre l'avortement et l'existence de l'hygroma.

L'hygroma peut être traité chirurgicalement, mais en prenant soin de stériliser le contenu au préalable par le formol et de provoquer la disjonction de la capsule conjonctive par une injection de sulfate de cuivre.

\section{SUMMARY}

Brucella Hygroma - The characteristic clinical feature of bovine brucellosis in Rwanda - Burundi.

Brucellosis exists all over Rwanda-Burundi varying between $1 \%$ and $20 \%$ of the cattle from one district to the next. The presence of a hygroma may be considered as positive evidence of the subcutaneous deposition of brucella infected material. Bacteriological and serological examination of the fluid obtained by puncture, will confirm the clinical diagnosis in $60 \%$ of cases. 
An examination of 1351 head of indigenous cattle belonging to native stockowners, 78 head showed hygroma of which 30 had from 2-8 separate swellings. Of the total of 133 hygroma counted in the 78 head, 60 were situated on the hind legs and 40 on the fore legs. The remaining 33 were recorded from a variety of sites such as the angle of the hip, the forehead, in the ligamentum nuchae and in the form of subcutaneous abscesses on the back.

In general the incidence was greater in aged cows but the existence of hygroma in heifers of 1-4 years was noted. They may appear at any time but usually after an abortion or parturition but were also noted in heifers prior to bulling.

A blood agglutination test may be negative in an animal with one or more brucella hygroma. The serum titre of agglutination may not correspond with that of the liquid of the hygroma. A relationship exists between abortion and the presence of hygroma.

An hygroma may be treated surgically but care should be taken to sterilise the contents in advance with formol and encourage the liberation of the conjunctive tissue capsule by an injection of copper sulphate.

\section{RESUMEN}

El higroma brucelósico : El aspecto clínoco característico de la brucelosis bovina en Ruanda-Burundi.

La brucelosis se extiende por todo el pais. Su porcentaje varía de una región a otra y oscila entre el 1 y 20 p. 200 . El higroma debe ser considerado sinónimo de la brucelosis en su manifestación subcutánea. Por el examen clínico donde predomina la prueba de la punción, y por el examen bacteriológico y serológico del líquido de punción, el diagnóstico es confirmado en un 60 p. 100 de casos. En una investigación sobre 1351 bóvidos de raza indígena de ganaderos nativos, 78 animales estón atacados de higroma y de ellos 48 presentan uno y 30 de dos a ocho. Sobre las 78 cabezas se cuentan 133 hygromas de los cuales 60 se encuentran en los miembros posteriores, 40 en los anteriores y 33 en lugares diversos como ángulo externo de la cadera, entre los ligamentos de la nuca y, bajo forma de abcesos subcutáneos, sobre el dorso.

Generalmente, las vacas de 7 a 10 años son las más frecuentemente atacadas, pero se han observado también sobre terneras de 1 a 4 años.

Los higromas aparecen en cualquier momento, pero generalmente tras un aborto o un parto. Sobre las terneras se obsevan ya antes de la pubertad. La aglutinación de sangre puede ser negativa sobre un animal con uno o varios higromas brucelósicos. El título de aglutinación del suero sanguíneo no se corresponde siempre con el de aglutinación de liquido del higroma. Existe una relation entre aborto y existensia de higroma.

El higroma puede tratarse quirúrgicamente, pero debe tomarse la precaución de esterilizur su contenido por el formol y de provocar la separación de la cápsula conjuntiva por una inyección de sulfato de cobre.

\section{BIBLIOGRAPHIE}

1. CAMARA A. (1948). - Bull. Serv. Elev. Ind. anim. A. O. F. 1948, I: 24.

2. SAQUET E. (1955). - Revue d'Elev. Méd. vét. Pays trop., 1955, $8: 5$.

3. PERREAU P. (1956). - Revue d'Eler. Méd. vét. Pays trop., 1956, $9: 247$.

4. BLANCHARD A. et SINALY COULIBALY (1954). - Revue d'Elev. Méd. vét. Poys trop., 1954, $7: 153$.
5. HENNING M. W. (1956). - Animal diseases in South Africa, 3 eedition, Ed. General News Agency Johannesburg.

6. HUTYRA - MAREK - MANNINGER - MOCSY (1959). - Spezielle Pathologie und Therapie der Haustiere, Fischer, édit., léna.

7. VANDER WATH J. G. (1939). - Journ. S. Afr. Vet. Med. Ass., 1939, 10 (3) : 91. 
8. BERGMAN A. M. et AGREN E. (1923). Skan. vet. Tidskr., 1923, $13: 18$.

9. BOYD W. L., DELEZ A. L., FITCH C. P. (1930). - Corn. Vot., 1930, 20: 263.

10. DAFAALA E. N. et KHAN A. Q. (1958). Bull. Epiz. Dis. Africa, 1958, $6: 243$.

11. DIETZ O., NAGEL E., TURICH K. (1959). - Tierârztl. Umsch., 1959, I4: 274.

12. THIENPONT D., WIKTOR T.; MORTELMANS J., VANDENABEELE G., BICHE Y., FAGARD P., PINCKERS F. R. (1958). Ann. Soc. belg. Méd. trop., 1958, 38 : 1049.

13. COYD C. R. et VAUGHAN L. C. (1957). J. Comp. Poth. 1957, $67: 53$.

14. KELLER H. (1951). - Der Lebensmitteltierárzt, 1951, 2 : 37.
15. HOFMANN W. (1952). - Dtsch. Tierärztl. Wschr., 1952, $59: 211$.

16. TUSZKIEWICZ A. R. ef SZEWCZYKOWSKI W. (1958). - Pr. Med. 1958, 66: 1343.

17. MANSON BAHR P. E. C. (1956). - J. trop. Med. Hyg. 1956, 59 : 103.

18. FAGARD P. et VAN BALEN (1961). - Communication personnelle.

19. SCHOENAERS F. (1950). - Ann. Méd. vét, 1950, $94: 174$.

20. Rapports annuels du Laboratoire vétérinaire d'Astrida, 1958, 1959; 1960.

21. ADAMANTIDIS D. (1956). - Bull. agric. Congo belge, 1956, 47 : 585.

22. MATHIEU P. (1960). - Bull. agric. Congo, 1960, $51: 885$. 